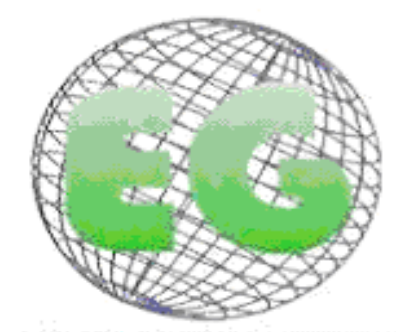

ISSN 1695-6141 N 27

\section{I}

-

\title{
Parto domiciliario planeado: asistido por enfermero obstetra
}

Parto domiciliar planejado: assistido por Enfermeiro Obstétra

\begin{abstract}
*Martins, CA., " ${ }^{* *}$ Almeida, NAM., ${ }^{* * *}$ de Mattos, DV.
*Enfermera Obstetra, Doctora en Enfermería, Profesora Asociada de la Facultad de Enfermería/Universidad Federal de Goiás - FEN/UFG. **Enfermera, Doctora en Ciencias de la Salud, Profesora Adjunta de la Facultad de Enfermería de la Universidad Federal de Goiás- FEN/UFG ${ }^{* * *}$ Enfermero, Supervisor de Prácticas en Obstetricia y Pediatría, Presidente de ABENFO-Goiás. E-mail: diegovmattos@hotmail.com Brasil.
\end{abstract}

Palabras clave: Parto em casa planeado; parto asistido; enfermero obstetra.

Palavras-chave: Parto domiciliar planejado; assistência ao parto; enfermeiro obstétra

Keywords: Planned home birth; assisted delivery; obstetric nurse

\section{RESUMEN}

En este artículo se reflexiona sobre el papel del enfermero obstetra en el parto en casa planeado. En este sentido, refleja los retos y las tendencias para la promoción y prestación de atención a la parturienta y al recién nacido. Señala el papel del enfermero obstetra en la asistencia planeada y el suministro y promoción de la calidad, eficacia y seguridad a las parturientas y al recién nacido como trípode de la humanización de la asistencia al parto y nacimiento.

\section{RESUMO}

Este artigo objetiva refletir acerca da atuação do enfermeiro obstetra no parto domiciliar planejado. Neste sentido, reflete os desafios e tendências para a provisão e promoção do cuidado à parturiente e ao recémnascido. Aponta o papel do enfermeiro obstetra na assistência planejada e a provisão e promoção da qualidade, efetividade e segurança às parturientes e ao recém-nascido como tripé da humanização da assistência ao parto e nascimento.

\section{ABSTRACT}

This article reflects on the obstetric nurse's role on the planned home childbirth. In this sense, it shows the challenges and trends for the promotion and the assistance of the parturient and new-born's care. It points out the obstetric nurse's role in the planned assistance, the supply and the promotion of quality, effectiveness and safety for the parturient and for the newborn as a tripod of humanization of the assistance to the delivery and the birth. 


\section{INTRODUCCIÓN}

La gestación, el parto y el puerperio son una de las experiencias humanas más importantes, con un fuerte potencial positivo y enriquecedor para todos los que participan. El embarazo y el parto son acontecimientos sociales que integran la experiencia reproductiva de hombres y mujeres. Es un proceso individual, una experiencia especial en el universo de la mujer y su pareja, que también involucra a sus familias y a la comunidad ${ }^{(1,16)}$.

El parto es una función de la mujer, mediante el cual los productos de la concepción (feto, líquido amniótico, placenta y membranas), son desplazados y expulsados del útero de manera fisiológica o mecánica ${ }^{(2)}$.

Es un proceso natural al que la mujer está sometida desde su existencia. Un momento de transición, en el que el cuerpo de la madre se prepara fisiológicamente durante el embarazo, para la expulsión del fruto de la concepción.

Se trata de un evento cultural que se inició con la existencia de la propia mujer, que se percibe como una purga en la mujer, que se describe en el Libro Bíblico del Génesis, cuando Eva, la primera mujer, conoció el pecado y escuchó de Dios, que iba a tener hijos por medio de dolores de parto ${ }^{(3)}$.

Con base en el conocimiento empírico, durante siglos el parto vaginal fue considerado un evento familiar, donde el acontecimiento era vivido por la familia, y el proceso de parto era llevado a cabo por las parteras, mujeres con una vida normal y conocimiento de la sociedad por su experiência ${ }^{(4)}$.

La hospitalización del parto en Brasil trajo un nuevo modelo asistencial y los avances tecnológicos que ahora contribuyen a disminuir las tasas de mortalidad materna y neonatal, promoviendo un aumento en el número de intervenciones durante el parto y nacimientos, así como partos con cesárea. Este enfoque del parto se ha convertido en un procedimiento electivo, dificultando la adopción de conductas de humanización del proceso natural del parto y nacimiento ${ }^{(5)}$.

Durante su trayectoria de vida la mujer durante el embarazo y parto pasa por diversas situaciones que favorecen las dudas y cambios de comportamiento. Por lo tanto, el parto es un acontecimiento de grandes cambios para la parturienta, por lo que la autonomía de la mujer y la decisión de la mujer sobre su cuerpo debe prevalecer en los procedimientos a $\operatorname{adoptar}^{(6)}$.

El embarazo y el parto son una de las experiencias humanas más importantes e impactantes para el cuerpo femenino, que puede tener una influencia positiva o negativa influenciando las experiencias futuras. Los acontecimientos que rodearon el proceso de parto y nacimiento en el ámbito hospitalario marcan una atmósfera peligrosa, sufrimiento, frustración de las expectativas, violencia física o simbólica, a menudo lo que hace difícil para las mujeres y sus familias convertir esa experiencia en algo positivo, gratificante y saludable (7).

Las acciones de humanización de la asistencia a la mujer en el proceso de parto tienden a una atención hacia la mujer y su familia en su unicidad, con necesidades específicas que van más allá de los aspectos biológicos y abarcan las circunstancias sociales, éticas, educativas y psicológicas presentes en las relaciones humanas ${ }^{(8)}$. 
Desde esta perspectiva, los aspectos emocionales del embarazo, el parto y puerperio son reconocidos ampliamente, creemos que la interacción de las mujeres embarazadas con los profesionales de la salud, especialmente de la enfermera obstétrica es esencial para ayudar a la parturienta permitiendo el proceso natural del parto.

En la actualidad, hay una discusión en la enfermería obstétrica acerca del retorno al parto domiciliario planeado, como instrumento relevante en la promoción y la humanización de la asistencia al parto y nacimiento.

Esa política avanza teniendo en cuenta la necesidad de dar concreción a los principios rectores del Sistema Único de Salud (SUS), en lo que se refiere a garantizar la calidad, efectividad y seguridad a la parturienta y al recién nacido durante el parto natural, el escenario natural del contexto domiciliario planificado.

Objeto de estudio: el nacimiento domiciliario planificado y sin distocia como forma de calidad de la asistencia humanizada al parto y nacimiento.

En esta comprensión, este artículo reflexiona sobre el papel de la enfermera obstétrica en el nacimiento domiciliario planificado.

Para desarrollar esta reflexión, buscamos profundizar teórico en la política de humanización de la atención al parto y nacimiento del Ministerio de Salud.

\section{ACTUACIÓN DE LA ENFERMERA OBSTÉTRICA EN EL PARTO DOMICILIARIO PLANIFICADO}

Las parteras tradicionales son responsables hoy en día del nacimiento de 450.000 niños por año en todo el mundo, lo que representa el $18 \%$ de los nacimientos del año(4).

La institucionalización del parto se produjo en los años 60, trayendo consigo un cambio considerable en este escenario, promoviendo una mejor calidad en la atención en el nacimiento con la introducción de un equipo multidisciplinar en las prácticas de atención al parto ${ }^{(4)}$.

Sin embargo, la alta tasa de intervenciones en el proceso fisiológico del parto no ha garantizado la calidad de la atención obstétrica ni la caída de los indicadores de morbilidad y mortalidad maternas. Esto representa un reto para todas las instituciones que atienden a las Mujeres durante el embarazo y el parto ${ }^{(1)}$.

El cambio del ambiente para el nacimiento, en Brasil, trajo consigo una serie de avances tecnológicos que contribuyeron a bajar las tasas de mortalidad materna y neonatal, trayendo además un aumento considerable en el número de intervenciones en el nacimiento, como el parto por cesárea. Este tipo de parto pasó a ser tratado como electiva obstaculizando el proceso de humanización en el nacimiento ${ }^{(9)}$.

El carácter no invasivo de las tecnologías de cuidado de la enfermería obstétrica reside en la creencia de que cuando el individuo establece un vínculo de confianza con el profesional, ambos comparten las decisiones en la planificación de sus cuidados ${ }^{(10)}$.

El enfermero es un profesional cualificado de forma técnica y científica para la asistencia al parto. Desde 1998, el Ministerio de Salud y sus secretarías iniciaron estrategias, estableciendo políticas centradas en la cualificación profesional de Enfermería y Obstetricia 
para actuar en la asistencia al parto vaginal. Financiación de la especialización en Obstetricia y ordenanzas para la inclusión del trabajo realizado por la enfermera en la tabla de pagos del Sistema Único de Salud (SUS), fueron algunas de las medidas desarrolladas por el Ministerio de Salud ${ }^{(11)}$.

En los últimos años hemos experimentado grandes cambios en la configuración de la atención obstétrica, en el que retornamos a los valores que van más allá de los aspectos científicos y tecnológicos, que apunta al rescate del modelo histórico de nacimiento, trayendo de vuelta el ambiente domiciliario como un lugar propicio para el parto. En este contexto, las enfermeras obstétricas sobresalen en el aumento del parto domiciliario planificado, buscando retomar la calidad de la atención al parto para la parturienta y al recién nacido ${ }^{(12)}$.

El actual modelo de prestación de atención implica prácticas culturales de comunidades remotas, con las parteras, pero se percibe una búsqueda por parte de la mujer embarazada por el parto ecológico (parto domiciliario planificado) en las principales ciudades de Brasil. La residencia es para estas madres, un ambiente seguro y factible para dar a luz ${ }^{(13)}$.

\section{PARTO DOMICILIARIO COMO UNA MEDIDA PARA PROMOVER LA CALIDAD Y SEGURIDAD PARA LAS PARTURIENTAS Y EL RECIÉN NACIDO}

La humanización de la asistencia prestada es condición esencial para que las acciones de salud se traduzcan en la resolución de los problemas identificados, en la satisfacción de las usuarias, en el fortalecimiento de las mujeres frente a la identificación de sus demandas, el empoderamiento de sus derechos buscando el auto-cuidado, la promoción y mejora en la calidad de vida - materna y neonatal ${ }^{(14)}$.

Las prácticas de obstetrícia, al desplegar sus prácticas en los servicios de salud, en la búsqueda de la humanización recomendada por la Organización Mundial de la Salud, pone a disposición de las mujeres embarazadas el conocimiento profesionales específico y cualificado, que se caracteriza por ser esencialmente relacional y deriva de un conocimiento estructurado en el servicio de enfermería.

Este conocimiento se aplica en sentido transversal, integrando saber popular y de varias disciplinas en la construcción de la atención. Por tener como herramientas básicas los cuerpos, que proporciona comodidad y autonomía al alentar a las mujeres a reconocer y desarrollar sus propias habilidades. Basándose en evidencias científicas, las enfermeras obstétricas comenzaron a utilizar técnicas que se consideran favorables para la evolución fisiológica del trabajo de parto y prácticas no farmacológicas en el alivio del dolor ${ }^{(15)}$.

Durante el parto, la mujer debe sentirse apoyado y segura, con el fin de minimizar los miedos y ansiedades. La valorización en las relaciones interpersonales y la confianza en el profesional que asiste a la parturienta promueve una sensación de seguridad a través de la atención recibida, por lo tanto, al sentirse cuidada en este momento la madre tendrá la sensación de que su hijo será bien cuidado.

La humanización de la atención del parto requiere, sobre todo, en lo que respecta a la ejecución de los aspectos biópsicosocioespirituales de la madre, acoger también a su familia como forma de ofrecer apoyo emocional durante el proceso que se vive, y permitir su comodidad y bienestar ${ }^{(9)}$. 


\section{CONSIDERACIONES FINALES}

Este artículo busca reflexionar sobre la práctica de la enfermería en la asistencia humanizada a la parturienta de alto y bajo riesgo. Aunque muchos estudios han puesto de relieve la importancia del parto domiciliario, sin distocias, como una medida de calidad, promoción y seguridad a las parturientas y al recién nacido de bajo riesgo.

De hecho, la eficacia de este tipo de parto domiciliario planificado asistido por profesionales médicos y, sobre todo enfermeros, es todavía muy incipiente. Pero, sin duda, su uso representa un gran avance en el cambio de paradigma de un hospital centrado en un enfoque natural, sin intervenciones innecesarias en el proceso del parto, en consonancia con la política de parto humanizado y nacimiento.

Por lo tanto, deben incluirse como rutina en la atención básica a parturientas de bajo riesgo obstétrico. Este entendimiento permitirá a los profesionales de la salud una actuación competente, humana, segura y asertiva en el parto domiciliario planificado.

Desde esta perspectiva, deben ser instituidas políticas para la capacitación de médicos y enfermeras con un estándar de calidad de la atención prestada a las mujeres en el proceso del parto, con servicios de apoyo en las instituciones de salud de referencia para la asistencia en los casos cuando esté indicado. Además de la atención integral a las mujeres requiere programas y servicios resolutivos, a fin de satisfacer la gran demanda de la población femenina.

Consideramos que estas reflexiones deben contribuir a que los gerentes de salud y profesionales del área llevenr a cabo acciones de promoción y prevención a las mujeres en el proceso del parto, como un derecho constitucional a la ciudadanía de la usuaria del Sistema Único de Salud y otros servicios de salud.

\section{REFERENCIAS}

1. Brasil. Ministério da Saúde. Secretaria de Atenção à Saúde. Departamento de Ações Programáticas Estratégicas. Área Técnica de Saúde da Mulher. Pré-natal e Puerpério: atenção qualificada e humanizada - manual técnico. Ministério da Saúde, Secretaria de Atenção à Saúde, Departamento de Ações Programáticas Estratégicas. Brasília (Brasil): Ministério da Saúde, 2005.

2. Rezende J, Montenegro CAB. Obstetrícia fundamental. $11^{\underline{a}}$ ed. Rio de Janeiro: Guanabara Koogan; 2008.

3. Teixeira NZF, Pereira WR. Parto hospitalar - experiências de mulheres da periferia de Cuiabá-MT. Rev. Bras de Enferm. v. 59, n. 6,p. 740-744, nov-dez, 2006.

4. Brenes AC. História da parturição no Brasil, Século XIX. Caderno de Saúde Pública. Rio de Janeiro, v. 7, n.2, 135-149, abr-jun, 1991.

5. BRASIL. Ministério da Saúde. Parto, aborto e puerpério. Assistência humanizada à mulher. Brasília (Brasil): Ministério da Saúde, 2003.

6. Oliveira SMJV, Riesco MLG, Miya CFR, Vidotto P. Tipo de parto: expectativas das mulheres. Rev. Latino-am enferm. São Paulo, v. 10, n. 5, p. 667-674, set-out, 2002.

7. Barros WLL, Costa E, Boeckmann LMM, et al. Parto Humanizado: Uma realidade na Casa de Parto? Rev Enferm UFPE [on line] 2011 jan./fev.5(1):67-74, ISSN:19818963.

Disponível:

http://www.ufpe.br/revistaenfermagem/index.php/revista/article/viewFile/1207/pdf 277, acessado em 20 de jun de 2011. 
8. Souza KRF, Dias MD. História oral: a experiência das doulas no cuidado à mulher. Acta Paul. Enferm. [online]. 2010, v.23, n.4, p. 493-499. ISSN 0103-2100. Disponível: http://www.scielo.br/pdf/ape/v23n4/08.pdf, acessado em 20 de jun de 2011.

9. Milbrath VM, Amestoy SC, Soares DC, Siqueira HCH. Vivências maternas sobre a assistência recebida no processo de parturição. Escola Anna Nery Rev Enferm [online] 2010, v.14, n.3, pp. 462-467. ISSN:1414-8145.Disponível: http://www.scielo.br/pdf/ean/v14n3/v14n3a05.pdf, acessado em 24 de jun de 2011.

10. Riesco MLG, Fonseca RMGS. Elementos constitutivos da formação e inserção de profissionais não-médicos na assistência ao parto. Caderno de Saúde Pública. Rio de Janeiro, v.18, n.3, p.685-698, mai-jul, 2002.

11. Vargens OMC, Progianti JM, Silveira ACF. O significado de desmedicalização da assistência ao parto no hospital: análise da concepção de enfermeiras obstétricas. Rev. Esc. Enferm. USP [online]. 2008,v.42,n.2,pp.339-346. ISSN 0080-6234. Disponível: http://www.scielo.br/pdf/reeusp/v42n2/a17.pdf, acessado em 5 de jun de 2011.

12. Campos SEV, Lana FCF. Resultados da assistência ao parto no Centro de Parto Normal Dr. David Capistrano da Costa Filho em Belo Horizonte, Minas Gerais, Brasil. Cad. Saúde Pública [online]. 2007, v.23, n.6, pp. 1349-1359. Disponível: http://www.scielo.br/pdf/csp/v23n6/09.pdf, acessado em 22 de jun de 2011.

13. Nascimento KC, Santos EKA, Erdmann AL, Júnior HJN, Carvalho, JN. A arte de partejar: experiência de cuidado das parteiras tradicionais de Envira/AM. Escola Anna Nery Rev. Enferm. [online] 2009 abr-jun; 13 (2):319-27.Disponível: http://www.eean.ufri.br/revista enf/20092/artigo\%2010.pdf, acessado em 30 de Jun de 2011.

14. Medeiros RMK, Santos IMM, Silva LR. A escolha pelo parto domiciliar: história de vida de mulheres que vivenciaram esta experiência. Escola Anna Nery Rev Enferm [online] 2008 dez; 12(4):765-72. Disponível: http://www.eean.ufri.br/revista enf/20084/20-ART\%20.pdf, acessado em 30 de jul de 2011.

15. Torres JA, Santos I, Vargens OMC. Construindo uma concepção de tecnologia de cuidado de enfermagem obstétrica: estudo sociopoético. Texto \& Contexto Enfermagem. 2008, 17(4):656-64. Disponível: www.scielo.br/pdf/tce/v17n4/05.pdf, acessado em 20 de jul de 2011.

16. Vasconcelos KL. PARTOGRAMA: aplicação de instrumento no processo parturitivo. Dissertação (Mestrado). 113p. Goiânia-Go. Programa de Pós-Graduação em Enfermagem, Faculdade de Enfermagem Universidade Federal de Goiás, 2009.

ISSN 1695-6141 